\title{
Cyclic-di-GMP Induces STING-Dependent ILC2 to ILC1 Shift During Innate Type 2 Lung Inflammation
}

\begin{abstract}
Kellen J. Cavagnero ${ }^{1,2}$, Jana H. Badrani ${ }^{1}$, Luay H. Naji ${ }^{1}$, Michael B. Amadeo ${ }^{1}$, Anthea S. Leng ${ }^{1}$, Lee Diego Lacasa ${ }^{1}$, Allyssa N. Strohm ${ }^{1}$, Samantha R. Renusch ${ }^{1}$, Suzanna S. Gasparian ${ }^{1}$ and Taylor A. Doherty ${ }^{1,3 *}$
\end{abstract}

${ }^{1}$ Department of Medicine, University of California, San Diego, La Jolla, CA, United States, ${ }^{2}$ Department of Dermatology, University of California, San Diego, La Jolla, CA, United States, ${ }^{3}$ Veterans Affairs San Diego Health Care System, La Jolla, $C A$, United States

Type 2 inflammation is found in most forms of asthma, which may co-exist with recurrent viral infections, bacterial colonization, and host cell death. These processes drive the accumulation of intracellular cyclic-di-nucleotides such as cyclic-di-GMP (CDG). Group 2 innate lymphoid cells (ILC2s) are critical drivers of type 2 lung inflammation during fungal allergen exposure in mice; however, it is unclear how CDG regulates lung ILC responses during lung inflammation. Here, we show that intranasal CDG induced early airway type 1 interferon (IFN) production and dramatically suppressed CD127+ST2+ ILC2s and type 2 lung inflammation during Alternaria and IL-33 exposure. Further, CD127-ST2-Thy1.2+ lung ILCs, which showed a transcriptomic signature consistent with ILC1s, were expanded and activated by CDG combined with either Alternaria or IL-33. CDG-mediated suppression of type 2 inflammation occurred independent of IL-18R, IL-12, and STAT6 but required the stimulator of interferon genes (STING) and type 1 IFN signaling. Thus, CDG potently suppresses ILC2-driven lung inflammation and promotes ILC1 responses. These results suggest potential therapeutic modulation of STING to suppress type 2 inflammation and/or increase anti-viral responses during respiratory infections.

Keywords: ILC, ILC1, ILC2, asthma, Cyclic-di-GMP, STING, COVID-19, innate lymphoid cell

\section{INTRODUCTION}

Group 2 innate lymphoid cells (ILC2s) were identified in 2010 and have since been recognized for their essential role in orchestrating innate type 2 immune responses in murine asthma models (1-3). Airway exposure to the clinically-relevant fungal allergen Alternaria alternata (Alt) promotes rapid epithelial cell IL-33 secretion and ILC2 production of IL-5 and IL-13, which drive peribronchial eosinophilic inflammation (4). ILC2s belong to a greater family of ILCs that includes ILC1s, which are involved in protective immune responses against intracellular pathogens. While ILC2s express the key transcription factor GATA-3 and produce type 2 cytokines, ILC1s express T-bet and produce interferon- $\gamma($ IFN $\gamma)$. 
Insights into asthma pathogenesis have revealed associations with bacterial colonization (5), viral infections (6), as well as inappropriate cell death and mitochondrial stress (7). These processes drive accumulation of cyclic-di-nucleotide danger signals inside of host cells (8). Free intracellular viral and host DNA are converted to cyclic-di-nucleotides by the host cytosolic enzyme cyclic-GMP-AMP-synthase (9). One particular cyclicdi-nucleotide, cyclic-di-GMP (CDG), is synthesized by bacteria ubiquitously as a secondary messenger (10). CDG is not host cell membrane permeable, suggesting CDG accumulation inside of host cells occurs by way of active transport $(11,12)$. CDG is unique to microbes yet binds numerous mammalian receptors including HCN (13), HpoR (14), Siderocalin (15), and the stimulator of interferon genes (STING) (16). Importantly, CDG has been shown to induce robust type 1 and type 3 interferons (IFN) that are critical for anti-viral responses (16-18).

Viral infections are a common cause of asthma exacerbations, though how type 1 and 3 IFNs contribute to asthma pathogenesis is unclear and may be different in specific asthma endotypes. Recently, the global SARS-CoV2 pandemic has led to rapid, broad interest in anti-viral and immunomodulatory strategies to prevent and treat severe disease. Studies in COVID-19 patients have shown that type 1 IFN responses are delayed and/or exaggerated at later phases of severe COVID-19 (19-22). Thus, strategies to control early airway infections through strong local anti-viral responses may be advantageous through modulation of type 1 and 3 IFN and ILC subsets. The effect of cyclicdi-nucleotides on lung ILC responses is unknown. Here, we investigated the role of CDG in fungal allergen and IL-33 driven asthma models.

\section{RESULTS}

\section{CDG Abrogates Alternaria-Induced ILC2-Driven Type 2 Airway Inflammation and Promotes Interferon Production}

To elucidate whether CDG regulates innate lung immunity, we utilized a well-described 3-day Alt-induced ILC2-driven model of eosinophilic airway inflammation (Figure 1A) (4). Intranasal CDG administration nearly abolished Alt-induced bronchoalveolar lavage fluid (BAL) and lung eosinophilia (Figures 1B,C, Supplementary Figure 1A). Concordantly, CDG significantly reduced BAL IL-5 and IL-13 levels (Figures 1D,E). Interestingly, CDG synergistically potentiated Alt-induced BAL and lung neutrophil accumulation (Figures 1F,G, Supplementary Figure 1B). In the absence of Alt, CDG did not have a significant effect on airway granulocytic infiltration (Figures 1B,C,F,G).

Recent studies have shown that ILC2 cytokine production is suppressed by multiple IFNs (23-25). Therefore, we next asked whether CDG drove IFN production during Alt exposure. Indeed, BAL IFN $\gamma$ (type 2 IFN) levels increased over 20 -fold on

Abbreviations: ILC, Innate lymphoid cell; ILC2, group 2 innate lymphoid cell; ILC1, group 1 innate lymphoid cell; Alt, Alternaria alternata; CDN, cyclic-dinucleotide; CDG, cyclic-di-GMP; STING, stimulator of interferon genes; IFN, interferon; BAL, bronchoalveolar lavage. average in mice receiving both Alt and CDG compared to Alt alone (Figure 1H). Further, BAL IFN $\beta$ (type 1 IFN) was increased 30 -fold $3 \mathrm{~h}$ following the first intranasal challenge in mice that received CDG (Figure 1I). BAL IFN $\lambda$ (type 3 IFN) levels were also higher in CDG treated mice at this early timepoint, though the difference was not significant (Supplementary Figure 1C).

To determine whether adaptive or innate lymphocytes, or both, were required for CDG-induced attenuation of type 2 inflammation, we performed experiments with Rag2-/- mice that possess ILCs (including NK cells) but are deficient in B and T cells as well as $I l 7 r-/$ - mice that lack both ILCs and the majority of T/B cells. Thus, the major difference between these strains is the presence of ILCs (26). No IL-5 and relatively few eosinophils were detectable in the airways of $I l 7 r-/$ - mice (Figures 1J,K, Supplementary Figure 1D). Following Alt challenge, lung eosinophil and BAL IL-5 levels were markedly increased in Rag2/- mice compared to $I l 7 r$-/- mice. As in wild type mice, CDG nearly abolished Alt-induced lung eosinophilia and reduced BAL IL-5 $(p=0.0518)$ in Rag2-/- mice. Collectively, these results both demonstrate that CDG combined with Alt induces a neutrophilic response associated with increased type 1 and 2 IFNs and suggest that CDG reduces ILC2-driven eosinophilic airway inflammation, independent of adaptive immunity.

\section{CDG Induces a Lung ILC2 to ILC1 Shift}

We next investigated the effect of CDG on ILC2 responses in the 3-day Alt model. Given the observed increase in BAL IFN $\gamma$, we also focused on changes in ILC1 responses after CDG exposure. ILC subsets are heterogeneous and show significant plasticity under different conditions $(4,27)$. Thus, prior to assessing the effect of in vivo CDG challenge on ILC subsets, we used transcriptomic analysis to validate the identity ILC1s and ILC2s following Alt administration. We reanalyzed our published RNAseq dataset of Alt challenged murine lung ST2+CD127+ and ST2-CD127- ILCs (Figure 2A) (4) and found that ST2+CD127+ ILCs highly expressed canonical ILC2 genes (Klrg1, Il7r, Areg, Il1rl1, and Gata3), whereas ST2CD127- ILCs highly expressed key ILC1 genes (Gzma, Gzmb, $\operatorname{Irf8}, \mathrm{Klrk1}$, and Klrb1) (Figure 2B). Furthermore, gene ontology pathway analysis indicated that one of the most differentially expressed pathways between ST2+CD127+ and ST2-CD127ILCs was IFN $\gamma$ production (Figure 2C). Thus, ST2+CD127+ and ST2-CD127- populations contain strong ILC2 and ILC1 signatures, respectively.

Intranasal CDG administration dramatically reduced the number of Alt-induced IL-5+ and IL-13+ lung ILC2s following ex vivo PMA/ionomycin stimulation (Figure 2D, Supplementary Figures 2A,B). Because of the relatively artificial nature of ex vivo stimulation, we next utilized Red5 IL-5 reporter mice and Smart13 IL-13 reporter mice to provide in vivo evidence of CDG-elicited ILC2 suppression $(28,29)$. Concordantly, we found that CDG attenuated Alt-induced lung ILC2 IL-5 and IL-13 production in vivo (Figures 2E,F). Contrary to ILC2 responses, the total number of IFN $\gamma+$ ILC1s was significantly increased following CDG challenge and ex vivo PMA/ionomycin stimulation (Figure 2G, Supplementary Figure 2C). Further, $\mathrm{BAL}$ IFN $\gamma$ levels were markedly increased in Rag2-/- mice 


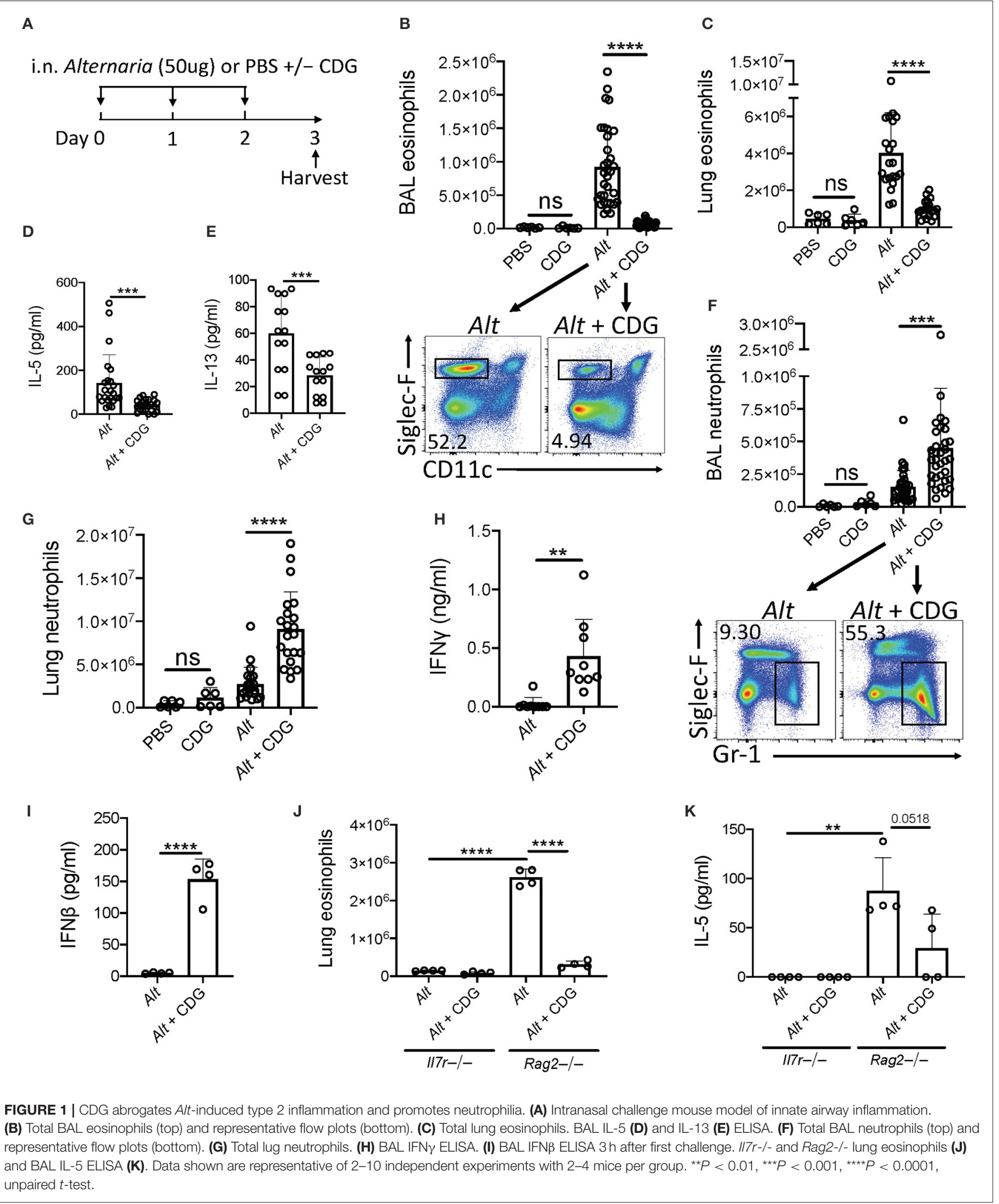

compared to Il7r-/- mice, suggesting that ILCs (and possibly $\mathrm{NK}$ cells) contribute significantly to CDG-induced IFN $\gamma$ secretion (Figure 2H).
In light of the CDG-induced shift from an ILC2 to ILC1 response and recent reports of ILC plasticity $(27,30)$, we further investigated the impact of CDG on ILC identity, activation, 


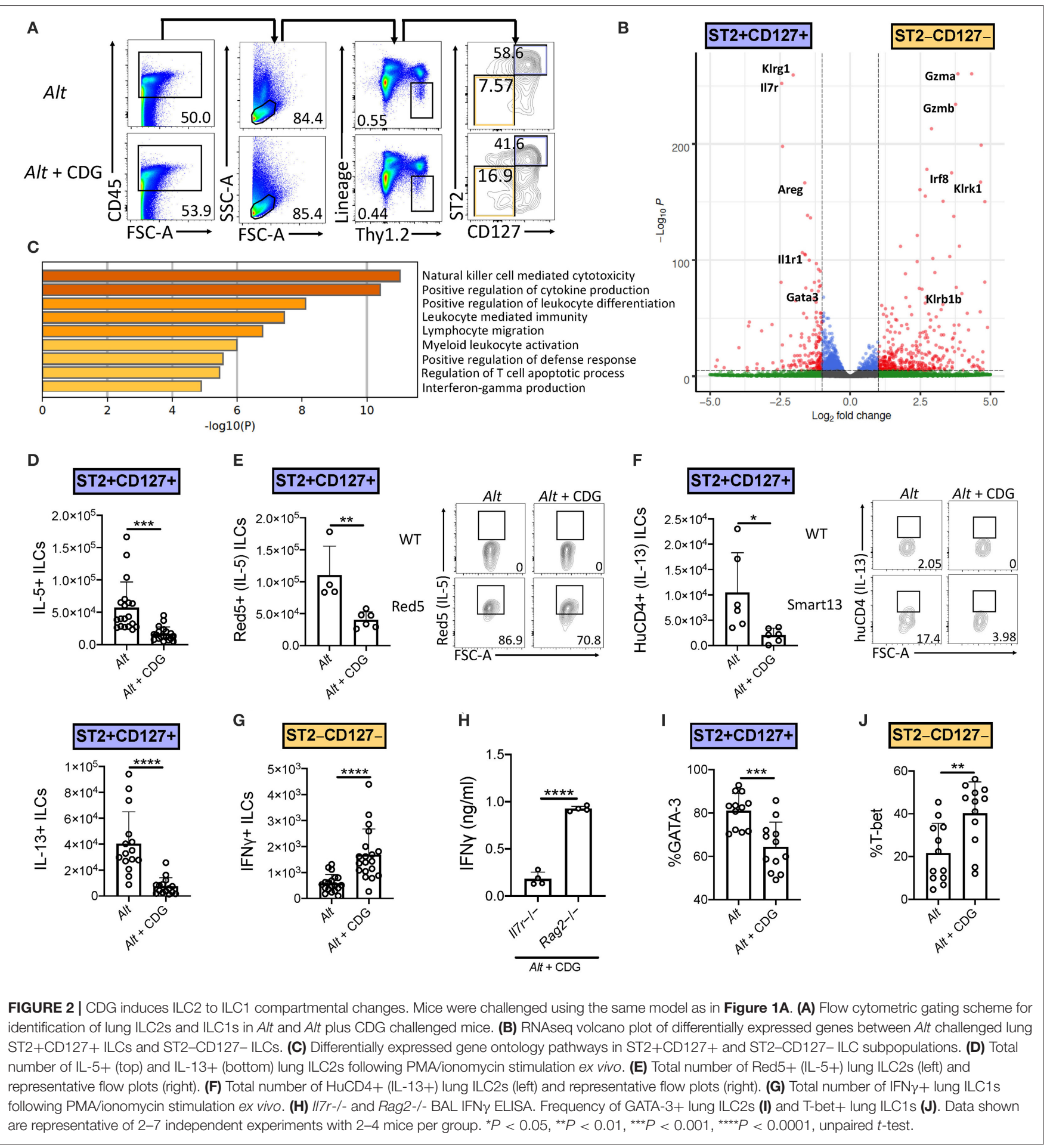

and proliferation. CDG decreased lung ILC2 expression of the canonical ILC2 master type 2 cytokine regulator GATA-3 (Figure 2I, Supplementary Figure 2D) and surface markers KLRG1 and ICOS (Supplementary Figures 2E,F). Conversely, CDG increased ILC1 expression of the master type 1 cytokine regulator T-bet by $20 \%$ on average (Figure 2J, Supplementary Figure 2G). Further, CDG attenuated ILC2 proliferation, as evidenced by decreased expression of Ki67 (Supplementary Figure 2H), but did not affect ILC2 activation status, as indicated by unchanged CD69 expression levels (Supplementary Figure 2I). Conversely, CDG did not affect ILC1 proliferation (Supplementary Figure 2J) but did significantly increase ILC1 activation status (Supplementary Figure 2K). Taken together, these findings 
indicate that CDG drives concomitant ILC2 suppression and ILC1 activation.

As conventional NK cells are also a source of IFN $\gamma$, we assessed numbers and activation of lung NK cells in the model. We found a similar increase in NK1.1+CD49b+CD3lymphocytes in the Alt, CDG, and Alt+CDG groups of mice compared to PBS treated mice (Supplementary Figure 3A). Further, there was no significant difference in NK cell IFN $\gamma$ expression between groups (Supplementary Figure 3B). Thus, IFN $\gamma+\mathrm{NK}$ cell do not appear to be differentially responsive after CDG+Alt exposure as compared with ST2-CD127-ILCs.

\section{CDG Induces ILC2 to ILC1 Shift During IL-33-Driven Type 2 Lung Inflammation}

We next asked whether CDG-induced ILC2 inhibition occurred downstream of IL-33 release. To that end, we challenged mice with exogenous IL-33 instead of Alt. Consistent with the Alt model, CDG severely attenuated IL-33-induced lung eosinophilia (Figure 3A) and robustly increased lung neutrophil accumulation (Figure 3B). Additionally, CDG administration markedly decreased BAL IL-5 (Figure 3C) and increased BAL IFN $\gamma$ by over 100-fold (Figure 3D). As with Alt, CDG decreased the total number of IL-33-induced IL-5+ and IL13+ lung ILC2s (Figures 3E,F, Supplementary Figures 4A,B) and increased the total number of IFN $\gamma+$ ILC1s (Figure 3G, Supplementary Figure 4C). Thus, CDG had effects on IL-33 driven lung responses comparable to those of the fungal allergen Alt, suggesting that CDG inhibition of ILC2 responses is not related to an effect on IL-33 levels during Alt exposure.

\section{ILCs Express STING but Are Not Directly Regulated by Extracellular CDG}

We next sought to determine whether ILCs are directly regulated by CDG in the presence of IL-33 activation. We first investigated whether ILCs express STING. Expression analysis indicated that along with lung macrophages and dendritic cells (DCs), ILCs highly expressed STING (Supplementary Figure 5A). We stimulated sort purified ILCs (Supplementary Figure 5B) with IL-33 and CDG for $48 \mathrm{~h}$. As expected, IL-33 increased IL-5 and IL-13 production (Supplementary Figures 5C,D). However, extracellular CDG had no effect on ILC type 2 cytokine production showing that ILCs are not directly suppressed by CDG despite expressing STING. CDG has been reported to directly activate phagocytic cells including dendritic, monocyte, and macrophage cell lines, suggesting that phagocytosis is a mechanism by which extracellular CDG accumulates intracellularly and can then activate STING (16). Importantly, these cells could produce IL-12, IL-18, or type 1 IFNs that could suppress ILC2s and promote ILC1 responses.

\section{IL-12 and IL-18R Are Dispensable for CDG-Induced Suppression of Type 2 Response}

The ILC2-driven Alt asthma model has been described as largely IL-33 dependent, though conversion of ST2+ ILC2s to ILC1s during lung inflammatory responses to influenza has been shown to be promoted by IL-12 and IL-18 $(31,32)$. Thus, we investigated whether IL-12 and IL-18 signaling were required for the observed granulocytic shift after addition of CDG to Alt. Surprisingly, we found that the levels of airway eosinophilia and neutrophilia, and the number of ILC2s and ILC1s, remained constant in mice lacking IL-12 (Figures 3H,I,L, Supplementary Figures 4H,I,M) and IL-18R (Figures 3J,K,M, Supplementary Figures 4J-L). Collectively, these results demonstrate that CDG induces ILC2 to ILC1 and eosinophil to neutrophil shifts independent of IL-12 and IL-18 signaling.

\section{CDG Suppression of Type 2 Inflammation and ILC2 Responses Is STING-Dependent}

Given the robust airway IFN accumulation induced by CDG, we next investigated whether STING (the stimulator of interferon genes) was required for suppression of type 2 lung inflammation and ILC2 responses. Strikingly, through comparison of wild type and STING deficient (Tmem173-/-) mice, we found that removal of STING completely rescued CDG-driven abrogation of Alt-induced lung eosinophilia and fully abrogated CDGinduced lung neutrophilia (Figures 4A,B). Moreover, lung ILC2 function was completely restored in the absence of STING (Figure 4C), and CDG-driven ILC1 IFN $\gamma$ induction was entirely STING-dependent (Figure 4D).

\section{STAT6 Is Not Required for Neutrophilic Airway Inflammation Induced by CDG}

A recent study demonstrated that STAT6 was required for STING-dependent chemokine production (33). Therefore, we sought to determine whether STAT6 was required for neutrophil accumulation in lung tissue following CDG challenge. Consistent with previous reports, Alt-induced eosinophilia was highly dependent upon STAT6 (Figure 4E) (26). Notably, we found that residual lung eosinophil levels following CDG administration were further reduced in the absence of STAT6. Unlike eosinophils, neutrophils accumulated in the airway independent of STAT6 (Figure 4F). These results demonstrate that while STAT6 regulates innate type 2 lung inflammation, likely through chemokine expression, it has no role in the STING-induced effects on neutrophil accumulation $(26,34)$.

\section{Type 1 IFN Signaling Is Indispensable for CDG-Induced Suppression of Type 2}

\section{Inflammation}

Type 1 IFNs are thought to be the major products of stimulator of interferon genes (STING) activation. To investigate whether type 1 IFN production was responsible for the observed CDG-induced immunomodulation, we compared type 1 IFN signaling deficient (Ifnar1-/-), wild type, and Tmem173-/- mice. Removal of type 1 IFN signaling rescued CDG-induced abrogation of BAL and lung eosinophilia (Figure 5A) but did not prevent CDG-induced BAL and lung neutrophilia (Figure 5B). Moreover, the decrease in total IL-13+ ILCs caused by CDG was dependent on type 1 IFN signaling (Figure 5C). Thus, type 1 IFNs are indispensable for CDG-mediated suppression of type 2 inflammation but not neutrophilia. These results are consistent with recent work demonstrating that type 1 IFNs suppress lung ILC2s during viral infection (24). 


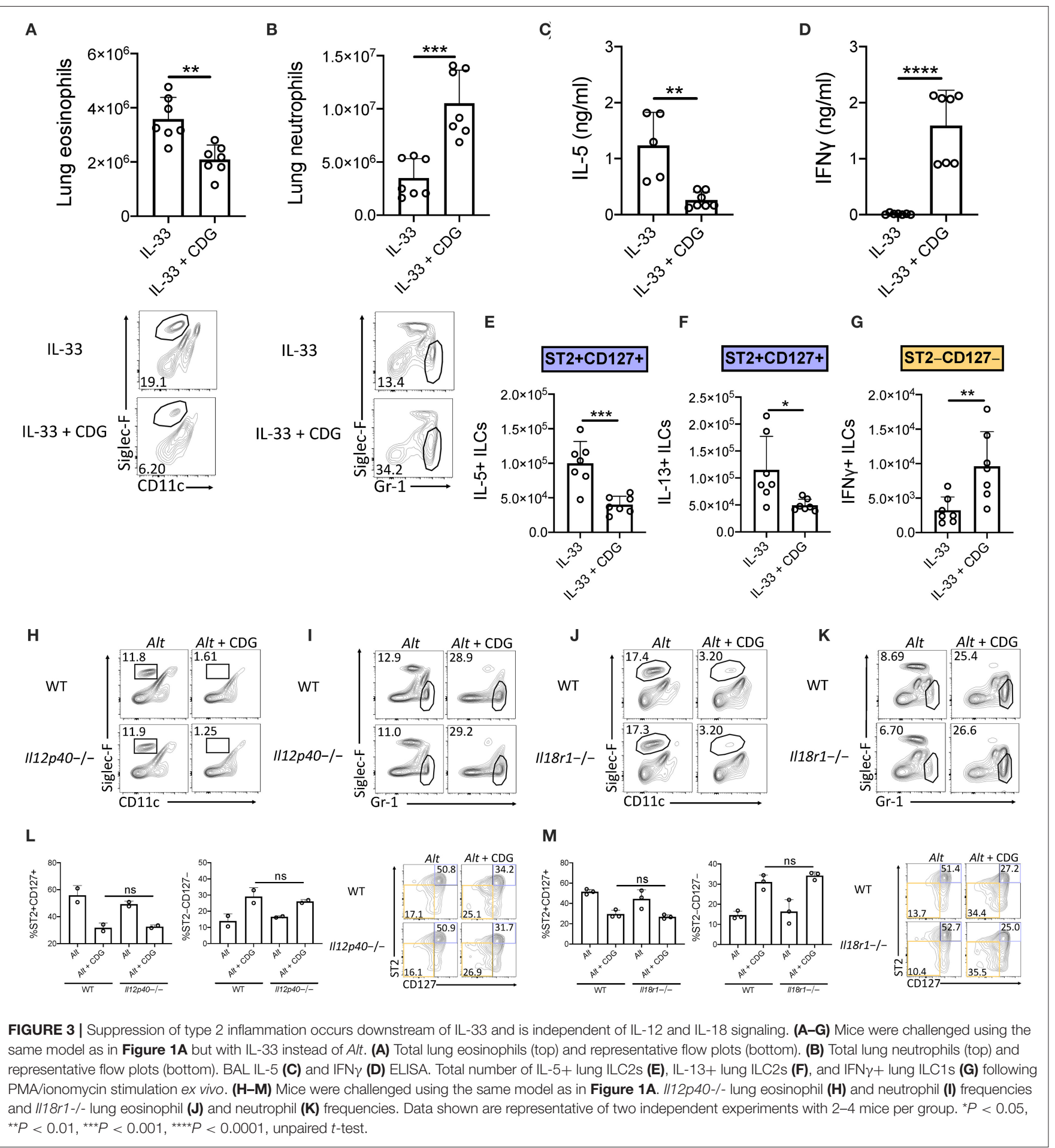

\section{IFN $\gamma$ Is Dispensable for CDG-Induced ILC and Granulocyte Changes}

Given that CDG elicited increases in airway IFN $\gamma$ that may also suppress ILC2 function $(23,25,35)$, we next investigated whether IFN $\gamma$ was required for CDG-induced lung granulocyte or ILC changes. IFN $\gamma$ monoclonal antibody blockade led to a modest non-significant increase in airway eosinophilia and IL-5+ ILC2s in mice challenged with Alt and CDG (Supplementary Figures 6A,C,E). Similar to type 1 IFN receptor deficient mice, inhibition of IFN $\gamma$ signaling had no effect on airway neutrophilia (Supplementary Figures 6B,D). These results support that IFN $\gamma$ is largely dispensable for CDG-induced immunomodulation of ILC2 responses and neutrophilia after exposure to $A l t+\mathrm{CDG}$. 


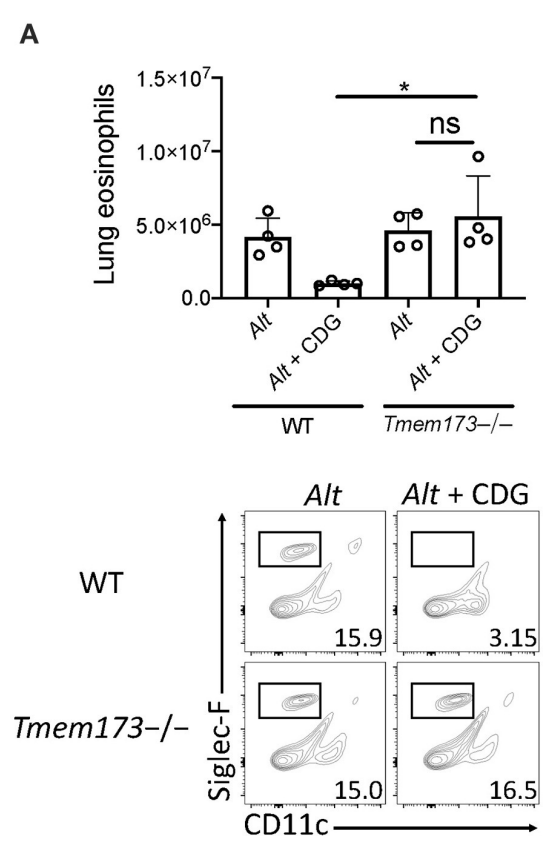

D
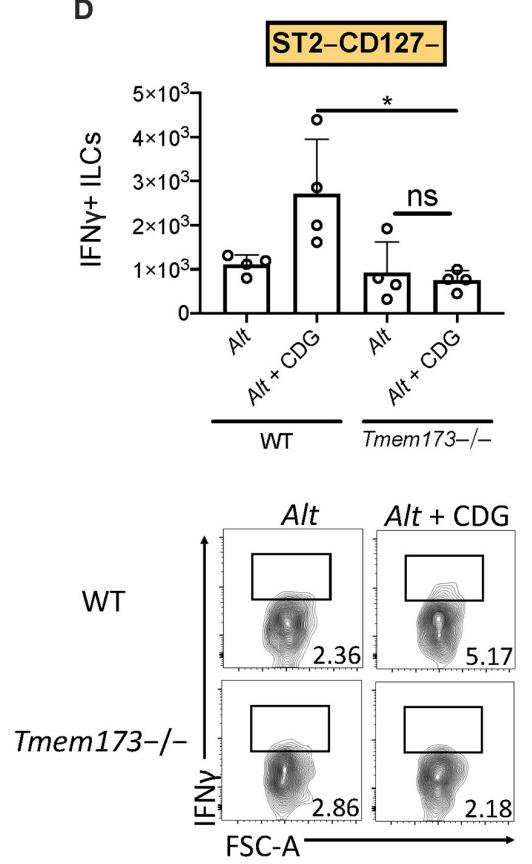

B
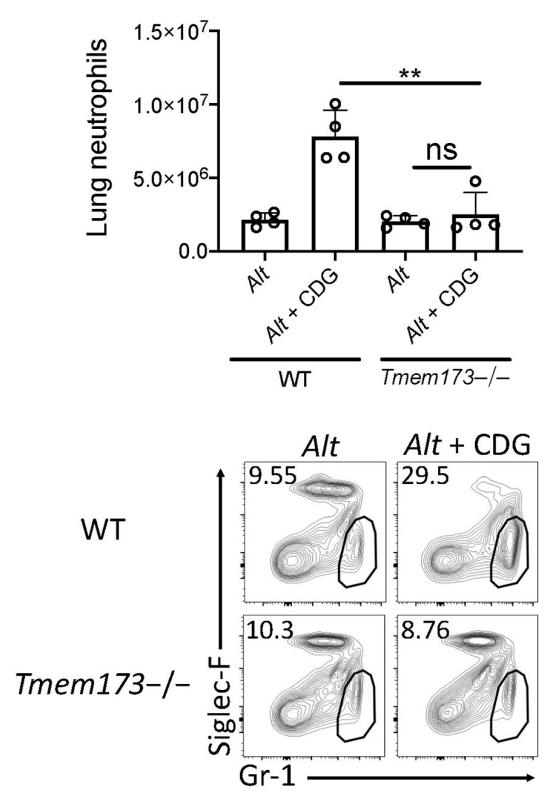

E
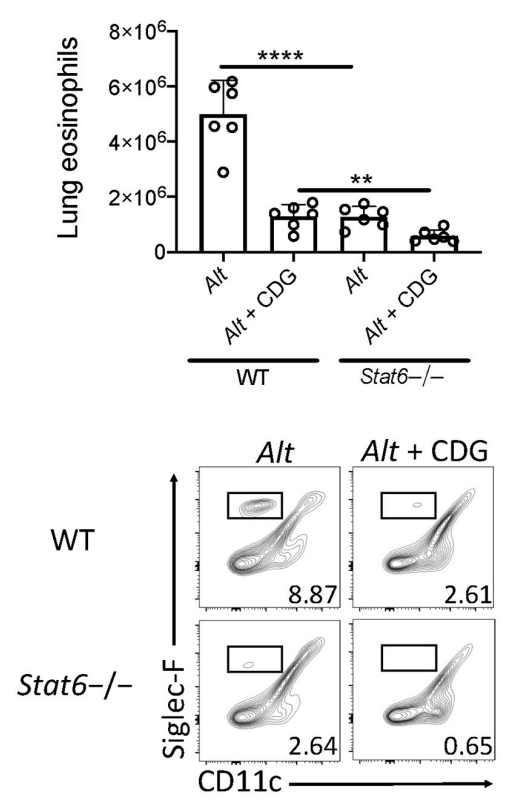

C
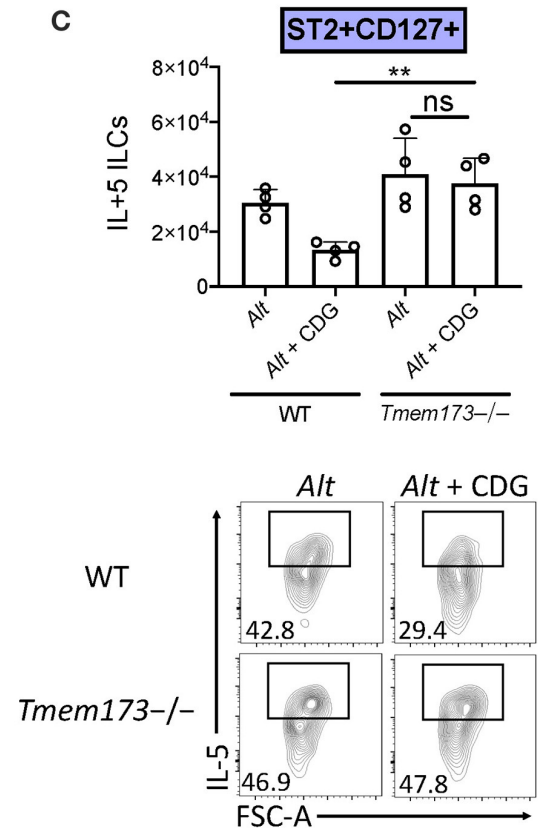

F
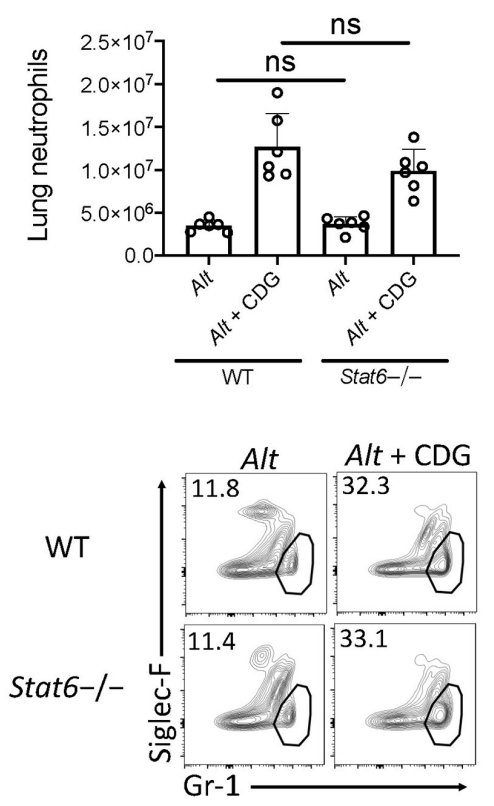

FIGURE 4 | CDG modulation of airway inflammation is STING-dependent and STAT6 independent. (A-D) Wild type and Tmem173-/- mice were challenged using the same model as in Figure 1A. (A) Total lung eosinophils (top) and representative flow plots (bottom). (B) Total lung neutrophils (top) and representative flow plots (bottom). (C) Total lung IL-5+ ILC2s (top) and representative flow plots (bottom). (D) Total lung IFN $\gamma+$ ILC1s (top) and representative plots (bottom). (E,F) Wild type and Stat6-/- mice were challenged using the same model as in Figure 1A. (E) Total lung eosinophils (top) and representative flow plots (bottom). (F) Total lung neutrophils (top) and representative flow plots (bottom). Data shown are representative of two independent experiments with $2-3$ mice per group. ${ }^{\star} P<0.05$, ${ }^{\star \star} P<0.01,{ }^{\star \star \star \star} P<0.0001$, unpaired $t$-test.

\section{DISCUSSION}

Asthma is largely a type 2 inflammatory airway disease and is associated with bacterial and viral infections, mitochondrial stress, and host cell death, which all lead to the accumulation of the danger-associated cyclic-di-nucleotides $(5-8,36)$. ILC2s promote type 2 inflammation in experimental asthma models and likely contribute to airway inflammation and 
A

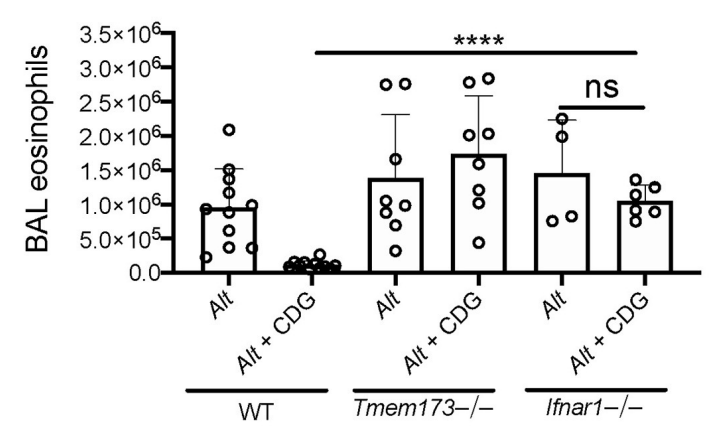

B

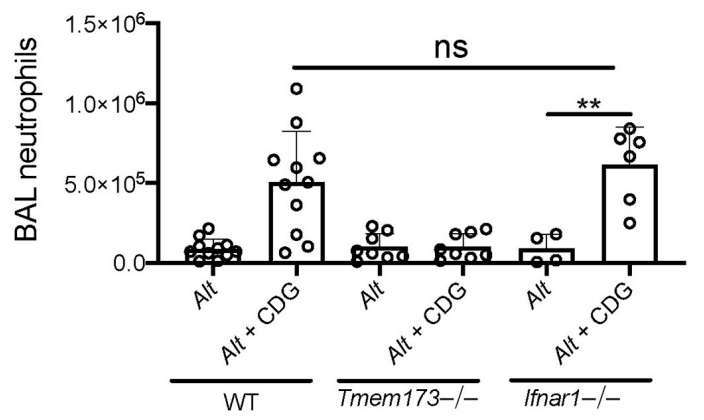

C

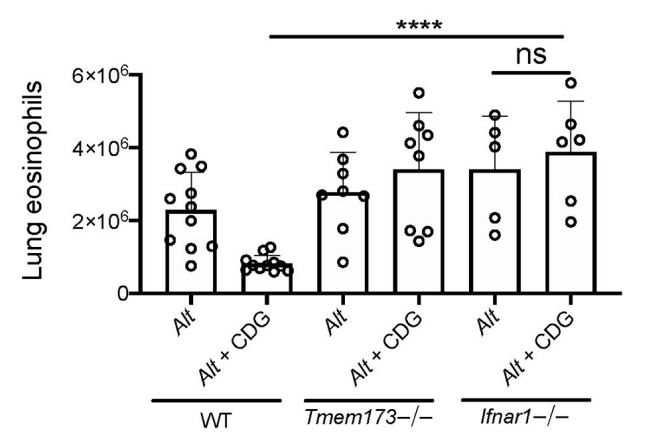

E

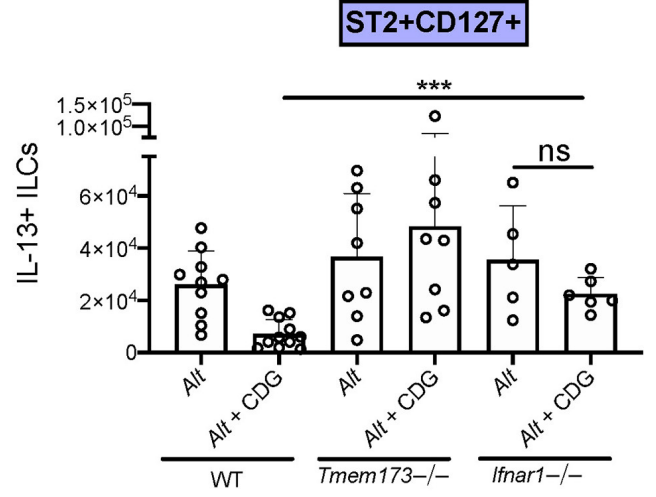

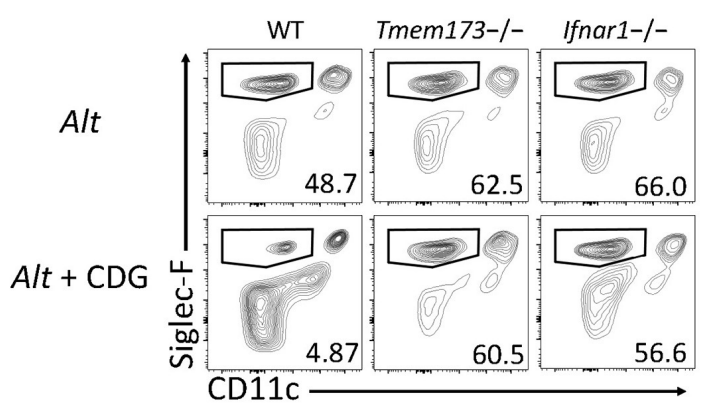

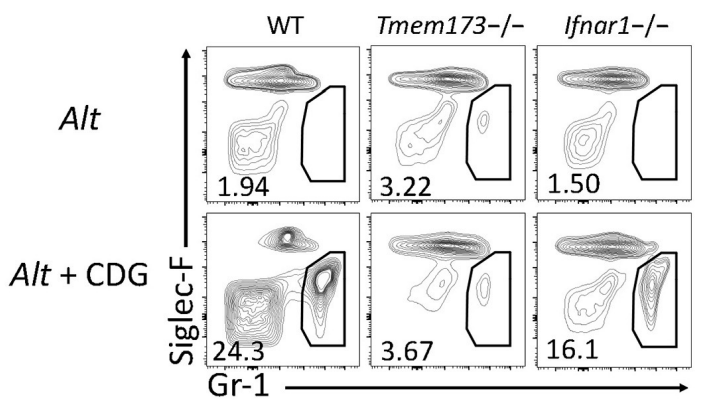

D
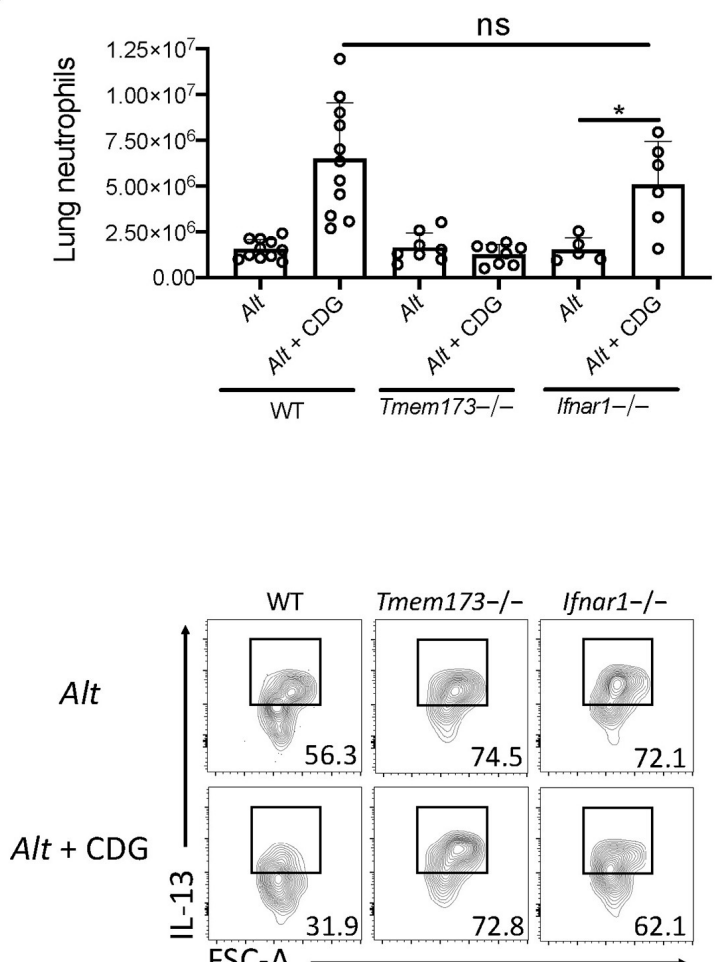

FIGURE 5 | Type 1 IFN signaling is required for CDG induced suppression of ILC2s and type 2 inflammation. Wild type, Tmem173-/-, and Ifnar1-/- mice were challenged using the same model as in Figure 1A. (A) Total number of BAL eosinophils (left) and representative plots (right). (B) Total number of BAL neutrophils (left) and representative flow plots (right). (C) Total number of lung eosinophils. (D) Total number of lung neutrophils. (E) Total number of IL-13+ST2+CD127+ ILC2s (left) and representative flow plots (right). Data shown are representative of 5 independent experiments with $1-3$ mice per group. ${ }^{\star} P<0.05,{ }^{* \star} P<0.01,{ }^{\star \star \star} P<0.001,{ }^{\star \star \star \star} P$ $<0.0001$, unpaired $t$-test. 
hyperresponsiveness in humans (37). Here, we investigated how in vivo administration of the cyclic-di-nucleotide CDG regulates ILC2s and innate type 2 inflammation. Our novel findings show that intranasal CDG challenge nearly abolished Alt-induced airway eosinophilia but promoted neutrophil accumulation. Congruently, CDG drove contraction of IL-5+ and IL-13+ lung ILC2s and expansion of IFN $\gamma+$ ILC1s. Mechanistically, we found that CDG modulated ILC responses downstream of Alt-induced IL-33 release and that the effect of CDG on airway type 2 inflammation was entirely STING- and type 1 IFN-dependent. Interestingly, the effect of CDG on airway neutrophilia was STING-dependent and type 1 IFN-independent. Thus, STING's involvement in pathogen responses, as well as activation by cytosolic mitochondrial DNA (from damaged mitochondria) and autophagy, could represent a critical, common pathway in the development of mixed airway inflammatory responses often found in asthma $(8,38-42)$.

While type 1 IFNs are the major products of STING activation, STING agonism has also been shown to induce the production of several inflammatory mediators including type 3 IFNs, IL-1 $\beta$, IL-6, TNF $\alpha$, CCL2, and CCL20 (43-46). In addition to elevated levels of type 1 IFNs, we observed increases in type 2 and 3 IFN following CDG administration, though the latter failed to reach significance. While type 1,2, and 3 IFNs have all been shown to inhibit ILC2 function and restrict type lung 2 inflammation (23-25, 47, 48), we found that only type 1 IFN was required for CDG-induced ILC2 suppression. Taken together with recent reports, our findings suggest a model in which intranasal CDG challenge drives STING-dependent early production of type 1 IFN by airway phagocytes, thereby suppressing ILC2-driven airway eosinophilia. Because the effect of CDG on Alt-induced neutrophilia we observed was independent of type 1 and 2 IFN signaling, non-IFN mediators downstream of STING likely contribute to CDG-induced neutrophil accumulation.

The effect of CDG on airway eosinophil and neutrophil levels we report here agrees with a previous report in which CDG was administered in an adaptive Th2 cell-driven chronic asthma model, though ILC changes were not assessed (49). Further, the ILC compartmental changes we observed mirror influenza virus induced ILC2 to ILC1 plasticity described in a recent study (32). However, that study demonstrated that IL-12 and IL-18 drove the attenuation of type 2 cytokine production, whereas we found that CDG abrogated type 2 inflammation independent of IL-12 and IL-18 signaling and dependent on STING and type 1 IFN. Collectively, these findings indicate that there are multiple ways to induce an ILC2 to ILC1 shift in the lungs.

The role of STING in ILC responses and lung inflammation we report here are novel and may be tissue specific. A recent investigation into the role of STING in the gastrointestinal mucosa revealed a nearly 2 -fold reduction in ILC2 frequency and IL-4 and IL-13 levels, and a 3-fold increase in ILC1 frequency, in STING deficient mice (50). Conversely, we found markedly increased airway ILC2s and type 2 cytokines, and decreased ILC1s and type 1 cytokines, in STING deficient mice following Alt and CDG challenge. Such highly divergent findings suggest the presence of distinct, tissue-specific mechanisms by which STING controls ILC responses. These results are consistent with recent work demonstrating other tissue specific ILC responses (51).

The mechanism by which CDG-induced STING activation regulates lung inflammation that we report here is also strikingly different from the mechanism by which a STING gain-offunction mutation was recently reported to regulate lung inflammation (52). The N153S STING gain-of-function mutation induced type $1 \mathrm{IFN}$-independent and $\mathrm{B}$ and $\mathrm{T}$ cell-dependent spontaneous lung disease, while we found that CDG induced type $1 \mathrm{IFN}$-dependent and $\mathrm{B} / \mathrm{T}$ cell-independent suppression of type 2 inflammation. These contrasting results suggest that the mechanism of STING activation governs the downstream signaling response, of which there are numerous.

The findings we report here might be leveraged for developing treatments to induce early, local control of respiratory viruses, including SARS-CoV-2, in which type 1 and 3 IFN control of virus may be critical for prevention of late complications in severe disease $(19,20)$. RNA viruses, such as coronaviruses, induce STING downstream of RIG-I and MAVS signaling (53). Importantly, however, coronaviruses have evolved mechanisms for inhibiting STING activation. For example, coronaviruses encode proteases that antagonize STING and prevent downstream IFN production $(54,55)$. Interestingly, bats, which harbor coronaviruses yet show no signs of immunopathology, constitutively express IFN and were recently shown to possess a distinct STING from that of other mammals (56). When bat STING protein was humanized with a single amino acid substitution, viral tolerance was lost, suggesting a critical role for STING activation and IFN expression in controlling viral replication. Additionally, patients with severe COVID-19 have impaired type 1 IFN responses (20), and early administration of the type 1 interferon IFN- $\alpha 2 b$ led to reduced in-hospital mortality in COVID-19 patients (21). Collectively, our work, coupled with these reports, suggests that CDG, or other human STING agonists, may be a potential low-cost, local respiratory therapeutic for early treatment of COVID-19 following SARS-CoV2 infection through broad generation of anti-viral type 1, 2, and 3 IFNs.

Our study is limited in that the mechanisms downstream of STING that account for airway neutrophil accumulation remain unclear. Consistent with previous reports, we showed that lung eosinophilia is dependent on STAT6 (26). A prior study showed that STAT6 was required for STING-mediated chemokine production and immune cell recruitment (33); however, in our study, CDG-induced airway neutrophil accumulation occurred independent of STAT6, which suggests the existence of an alternative pathway by which immune cells accumulate in the presence of STING agonism. Further, we cannot exclude additional cell types such as NK cells as important sources of IFN $\gamma$ during CDG exposure. Future studies investigating these mechanisms are warranted.

In summary, our work demonstrates that CDG drives STING-dependent IFN production, ILC1 activation and accumulation, as well as ILC2 suppression and abrogation of innate type 2 innate airway inflammation. The knowledge set forth in this report is critical for understanding how allergic airway disease pathogenesis may be impacted by lung insults 
due to cellular stress, bacterial infection, or viral infections such as SARS-CoV2.

\section{MATERIALS AND METHODS}

\section{Mice}

6- to 12-week-old female C57BL/6 WT mice were obtained from The Jackson Laboratory (Bar Harbor, Me). Rag2-/-, Il7r-/-, Il12p40-/-, Ifnar1-/-, Tmem173-/-, and Stat6-/- mice were obtained from The Jackson Laboratory and bred in-house. Red5 mice were obtained from Dr. Nunzio Bottini (UCSD), originated from Dr. Richard Locksley (UCSF), and bred inhouse (29). Smart13 mice were obtained from The Jackson Laboratory, originated from Dr. Richard Locksley (UCSF), and bred in-house (28). Il18r1-/- mice were obtained from Dr. Hal Hoffman (UCSD) and bred in-house. All animal experiments were approved by the University of California, San Diego Institutional Animal Care and Use Committee.

\section{In vivo Alternaria and CDG Models}

WT, gene knockout, and cytokine reporter mice were challenged intranasally with PBS, 5 ug CDG, 50 ug of Alt extract (Greer, lot number 299382), or $5 \mathrm{ug}$ CDG and $50 \mathrm{ug}$ Alt extract in 40 $\mathrm{uL}$ every $24 \mathrm{~h}$ for 3 days and euthanized $24 \mathrm{~h}$ following the third challenge for BAL collection and lung tissue harvest. A subset of experiments was performed with only the latter two experimental groups. Further, in select experiments, Alt was replaced with exogenous IL-33 (500 ng). Additionally, a subset of experiments was performed with intraperitoneal IFN $\gamma$ blocking antibody or isotype (333 ug, BioXCell) injections on D-1, D0, and D1. For experiments in which ILCs were isolated, mice were challenged with Alt four times over 10 days to expand the ILC population. For experiments investigating early cytokine release, mice were euthanized $3 \mathrm{~h}$ following the first challenge. BAL was performed with $2 \% \mathrm{BSA}$ in PBS; the first draw was $500 \mathrm{uL}$ and draws 25 were $600 \mathrm{uL}$. BAL was centrifuged at $1,500 \mathrm{rpm}$ for $5 \mathrm{~min}$ at $4^{\circ} \mathrm{C}$ and the supernatant was stored at $-20^{\circ} \mathrm{C}$ for ELISA and cells were counted and phenotyped using flow cytometry. Lungs were digested using the Mouse Lung Dissociation Kit (Miltenyi Biotec) according to the manufacturer's protocol, filtered with a $40 \mathrm{um}$ mesh, and cells were counted and phenotyped using flow cytometry.

\section{Flow Cytometry}

Mouse BAL and lung cells were suspended in a solution of $2 \%$ FBS and $0.01 \%$ sodium azide in PBS and counted on a Novocyte (Acea Biosciences). Cells were incubated with an unconjugated $\mathrm{mAb}$ to $\mathrm{CD} 16 / \mathrm{CD} 32$ for $10 \mathrm{~min}$ at $4^{\circ} \mathrm{C}$ to block non-specific $\mathrm{Fc}$ receptor binding and then incubated for $30 \mathrm{~min}$ with fluorochrome conjugated antibodies at $4^{\circ} \mathrm{C}$. All antibodies were purchased from BioLegend unless otherwise noted. To identify eosinophils (CD45+Siglec-F+CD11c-) and neutrophils (CD45+GR-1+Siglec-F-), BAL or lung cells, or both, were stained with PerCP-conjugated anti-CD45.2, PE-conjugated anti-Siglec-F (BD), FITC-conjugated antiCD11c, and APC-conjugated anti-GR-1. For the identification of ILCs (CD45.2+lineage-Thy1.2+ lymphocytes), ILC2s
(CD45.2+lineage- Thy1.2+ST2+CD127+), and ILC1s (CD45.2+lineage-Thy1.2+ST2-CD127-) lung cells were incubated with FITC-conjugated lineage cocktail (anti-CD3e, anti-GR-1, anti-CD11b, anti-B220, anti-Ter119), anti-CD11c, anti-NK1.1, anti-CD5, anti-FcER1, anti-TCR $\beta$, and antiTCR $\gamma \delta$; PerCP-conjugated anti-CD45.2; eFluor 450-conjugated anti-Thy1.2 (ThermoFisher); APC-conjugated anti-ST2; and PE-Cy7-conjugated anti-CD127. In select experiments, ILCs were also stained with PE-conjugated anti-CD69, anti-ICOS, or anti-KLRG1. For the identification of NK cells (CD3CD49b+NK1.1+ lymphocytes), lung cells were incubated with PerCP-conjugated anti-CD45.2, APC-conjugated anti-CD3, PE-conjugated anti-CD49b, and FITC-conjugated anti-NK1.1.

For transcription factor and STING staining, surface-stained cells were fixed and permeabilized with an intracellular staining kit (ThermoFisher) according to the manufacturer's protocol and stained for $30 \mathrm{~min}$ at $4^{\circ} \mathrm{C}$ with PE-conjugated anti-GATA-3 (ThermoFisher), anti-Ki67 (ThermoFisher), anti-T-Bet, or antiSTING (Sigma).

For ILC and NK cytokine staining, lung cells from WT and knockout mice were incubated for $3 \mathrm{~h}$ at $37^{\circ} \mathrm{C}$ with a phorbol 12-myristate 13-acetate, ionomycin, brefeldin $\mathrm{A}$, and monensin cell stimulation cocktail (ThermoFisher) in RPMI 1640 media supplemented with penicillin/streptomycin, $10 \% \mathrm{FBS}$, glutamine, and 2-mercaptoethanol (ThermoFisher). Stimulated cells were surfaced stained for ILC2s and ILC1s as described above, fixed and permeabilized with an intracellular cytokine staining kit (BD) according to the manufacturer's protocol, and stained for 30 min at $4{ }^{\circ} \mathrm{C}$ with PE-conjugated anti-IL-5 or anti-IL-13 and APC-Cy7-conjugated anti-IFN $\gamma$.

Reporter mice were used to assess cytokine staining directly ex vivo without stimulation. To visualize in vivo IL-13 in Smart13 mice, human CD4 was stained with PE-conjugated anti-human CD4 (28). To visualize in vivo IL-5 in Red5 mice, the PE channel was used without any requisite staining (29). ILCs were stained with DAPI (ThermoFisher) to discriminate live and dead cells. Finally, samples were analyzed with a Novocyte (Acea Biosciences) flow cytometer or sorted for in vitro stimulation experiments with a FACSAria II (BD) at the UCSD Human Embryonic Stem Cell Core Facility.

\section{In vitro Stimulation}

Sort purified ILCs were first allowed to rest in vitro for $48 \mathrm{~h}$ with $10 \mathrm{ng} / \mathrm{ml}$ IL-2 and IL-7. Following media change, ILCs were cultured with either IL-2 $(10 \mathrm{ng} / \mathrm{ml})$ and IL-7 $(10 \mathrm{ng} / \mathrm{ml})$; IL$2(10 \mathrm{ng} / \mathrm{ml})$, IL-7 (10 ng/ml), CDG $(10 \mu \mathrm{M}) ; \mathrm{IL}-2(10 \mathrm{ng} / \mathrm{ml})$, IL-7 (10 ng/ml), and IL-33 (30 ng/ml); or IL-2 (10 ng/ml), IL-7 $(10 \mathrm{ng} / \mathrm{ml})$, IL-33 $(30 \mathrm{ng} / \mathrm{ml})$, and CDG $(10 \mu \mathrm{M})$. After $24 \mathrm{~h}$ of stimulation, supernatants were collected for ELISA.

\section{ELISA}

ELISAs for IL-5 (R\&D), IL-13 (R\&D), IFN $\gamma$ (ThermoFisher), IFN $\beta$ (R\&D), and IFN $\lambda(R \& D)$ were performed on BAL supernatants according to the manufacturers' protocols. ELISA plates were read on a model 680 microplate reader (Bio-Rad) at $450 \mathrm{~nm}$. 


\section{RNAseq}

Publicly available RNA sequencing of mouse lung ILC2s and ILC1s (GEO: GSE136156) was downloaded. Reads were then aligned to reference genome mm10 using TopHat. DUST scores were calculated with PRINSEQ Lite and low complexity reads (DUST > 4) were removed from the BAM files. Read counts to each genomic feature are obtained with the HTSeq count program. Differentially expressed genes were identified with DESeq2 and pathway analysis was performed with Metascape.

\section{Data Analysis and Statistics}

Flow cytometry data were analyzed using FlowJo version 10.4.1 (Flowjo). For all experiments (except RNAseq), statistical analysis was performed using GraphPad Prism software (GraphPad) using unpaired $t$-tests (2-tailed). $P$-values of $<0.05$ were considered statistically significant.

\section{DATA AVAILABILITY STATEMENT}

The datasets presented in this study can be found in online repositories. The names of the repository/repositories and accession number(s) can be found in the article/Supplementary Material.

\section{ETHICS STATEMENT}

The animal study was reviewed and approved by UC San Diego IACUC.

\section{REFERENCES}

1. Moro K, Yamada T, Tanabe M, Takeuchi T, Ikawa T, Kawamoto H, et al. Innate production of $\mathrm{T}(\mathrm{H}) 2$ cytokines by adipose tissue-associated c-Kit $(+) \mathrm{Sca}-1(+)$ lymphoid cells. Nature. (2010) 463:540-4. doi: 10.1038/nature08636

2. Neill DR, Wong SH, Bellosi A, Flynn RJ, Daly M, Langford TK, et al. Nuocytes represent a new innate effector leukocyte that mediates type-2 immunity. Nature. (2010) 464:1367-70. doi: 10.1038/nature08900

3. Price AE, Liang HE, Sullivan BM, Reinhardt RL, Eisley CJ, Erle DJ, et al. Systemically dispersed innate IL-13-expressing cells in type 2 immunity. Proc Natl Acad Sci USA. (2010) 107:11489-94. doi: 10.1073/pnas.1003988107

4. Cavagnero KJ, Badrani JH, Naji LH, Amadeo MB, Shah VS, Gasparian S, et al. Unconventional ST2- and CD127-negative lung ILC2 populations are induced by the fungal allergen Alternaria alternata. J Allergy Clin Immunol. (2019) 144:1432-5.e1439. doi: 10.1016/j.jaci.2019.07.018

5. Zhang Q, Illing R, Hui CK, Downey K, Carr D, Stearn M, et al. Bacteria in sputum of stable severe asthma and increased airway wall thickness. Respir Res. (2012) 13:35. doi: 10.1186/1465-9921-13-35

6. Holtzman MJ. Asthma as a chronic disease of the innate and adaptive immune systems responding to viruses and allergens. J Clin Invest. (2012) 122:2741-8. doi: $10.1172 /$ JCI60325

7. Lambrecht BN, Hammad H. Death at the airway epithelium in asthma. Cell Res. (2013) 23:588-9. doi: 10.1038/cr.2013.26

8. Chen Q, Sun L, Chen ZJ. Regulation and function of the cGAS-STING pathway of cytosolic DNA sensing. Nat Immunol. (2016) 17:1142-9. doi: 10.1038/ni.3558

9. Ryan RP, Fouhy Y, Lucey JF, Dow JM. Cyclic di-GMP signaling in bacteria: recent advances and new puzzles. J Bacteriol. (2006) 188:8327-34. doi: 10.1128/JB.01079-06

10. Burdette DL, Monroe KM, Sotelo-Troha K, Iwig JS, Eckert B, Hyodo M, et al. STING is a direct innate immune sensor of cyclic di-GMP. Nature. (2011) 478:515-8. doi: 10.1038/nature10429

\section{AUTHOR CONTRIBUTIONS}

KC, JB, MA, LN, LL, AL, AS, SR, and SG performed the experiments. $\mathrm{KC}$ and $\mathrm{TD}$ designed and interpreted the experiments and wrote the manuscript. All authors contributed to the article and approved the submitted version.

\section{FUNDING}

KC was supported by NIH grant T32 DK007202. TD was supported by Veterans Affairs BLR\&D Award BX005073 and NIH grants AI 114585 and AI 070535.

\section{ACKNOWLEDGMENTS}

We thank Dr. Hal Hoffman and Dr. Nunzio Bottini for the Il18r1-/- and Red5 mice, respectively. We would also like to thank Cody Fine and Jesus Olvera of the UCSD Human Embryonic Stem Cell Core for help with cell sorting as well as bioRxiv for hosting a preprint of this manuscript (57).

\section{SUPPLEMENTARY MATERIAL}

The Supplementary Material for this article can be found online at: https://www.frontiersin.org/articles/10.3389/fimmu. 2021.618807/full\#supplementary-material
11. Kumagai Y, Matsuo J, Hayakawa Y, Rikihisa Y. Cyclic di-GMP signaling regulates invasion by Ehrlichia chaffeensis of human monocytes. J Bacteriol. (2010) 192:4122-33. doi: 10.1128/JB.00132-10

12. Petersen E, Mills E, Miller SI. Cyclic-di-GMP regulation promotes survival of a slow-replicating subpopulation of intracellular Salmonella Typhimurium. Proc Natl Acad Sci USA. (2019) 116:6335-40. doi: 10.1073/pnas.1901 051116

13. Lolicato M, Bucchi A, Arrigoni C, Zucca S, Nardini M, Schroeder $\mathrm{I}$, et al. Cyclic dinucleotides bind the C-linker of HCN4 to control channel cAMP responsiveness. Nat Chem Biol. (2014) 10:457-62. doi: $10.1038 /$ nchembio. 1521

14. Li W, Li M, Hu L, Zhu J, Xie Z, Chen J, et al. HpoR, a novel c-di-GMP effective transcription factor, links the second messenger's regulatory function to the mycobacterial antioxidant defense. Nucl Acids Res. (2018) 46:3595-611. doi: 10.1093/nar/gky146

15. Li W, Cui T, Hu L, Wang Z, Li Z, He ZG. Cyclic diguanylate monophosphate directly binds to human siderocalin and inhibits its antibacterial activity. Nat Commun. (2015) 6:8330. doi: 10.1038/ncomms9330

16. Cui T, Cang H, Yang B, He ZG. Cyclic dimeric guanosine monophosphate: activation and inhibition of innate immune response. J Innate Immun. (2019) 11:242-8. doi: 10.1159/000492679

17. Blaauboer SM, Mansouri S, Tucker HR, Wang HL, Gabrielle VD, Jin L. The mucosal adjuvant cyclic di-GMP enhances antigen uptake and selectively activates pinocytosis-efficient cells in vivo. Elife. (2015) 4:17. doi: 10.7554/eLife.06670.017

18. Mcwhirter SM, Barbalat R, Monroe KM, Fontana MF, Hyodo M, Joncker NT, et al. A host type I interferon response is induced by cytosolic sensing of the bacterial second messenger cyclic-di-GMP. J Exp Med. (2009) 206:1899-911. doi: $10.1084 /$ jem.20082874

19. Blanco-Melo D, Nilsson-Payant BE, Liu WC, Uhl S, Hoagland D, Moller $\mathrm{R}$, et al. Imbalanced host response to SARS-CoV-2 drives development of COVID-19. Cell. (2020) 181:1036-45 e1039. doi: 10.1016/j.cell.2020.04.026 
20. Gruber C. Impaired interferon signature in severe COVID-19. Nat Rev Immunol. (2020) 20:353. doi: 10.1038/s41577-020-0 335-0

21. Wang N, Zhan Y, Zhu L, Hou Z, Liu F, Song P, et al. Retrospective multicenter cohort study shows early interferon therapy is associated with favorable clinical responses in COVID-19 patients. Cell Host Microbe. (2020) 28:45564.e2. doi: 10.1016/j.chom.2020.07.005

22. Zhou Z, Ren L, Zhang L, Zhong J, Xiao Y, Jia Z, et al. Heightened innate immune responses in the respiratory tract of COVID-19 patients. Cell Host Microbe. (2020) 27:883-90 e882. doi: 10.1016/j.chom.2020.04.017

23. Molofsky AB, Van Gool F, Liang HE, Van Dyken SJ, Nussbaum JC, Lee J, et al. Interleukin-33 and interferon- $\gamma$ counter-regulate group 2 innate lymphoid cell activation during immune perturbation. Immunity. (2015) 43:161-74. doi: 10.1016/j.immuni.2015.05.019

24. Duerr CU, Mccarthy CD, Mindt BC, Rubio M, Meli AP, Pothlichet J, et al. Type I interferon restricts type 2 immunopathology through the regulation of group 2 innate lymphoid cells. Nat Immunol. (2016) 17:65-75. doi: 10.1038/ni.3308

25. Califano D, Furuya Y, Roberts S, Avram D, Mckenzie ANJ, Metzger DW. IFN- $\gamma$ increases susceptibility to influenza A infection through suppression of group II innate lymphoid cells. Mucosal Immunol. (2018) 11:209-19. doi: $10.1038 / \mathrm{mi} .2017 .41$

26. Doherty TA, Khorram N, Chang JE, Kim HK, Rosenthal P, Croft M, et al. STAT6 regulates natural helper cell proliferation during lung inflammation initiated by Alternaria. Am J Physiol Lung Cell Mol Physiol. (2012) 303:L57788. doi: 10.1152/ajplung.00174.2012

27. Krabbendam L, Bal SM, Spits H, Golebski K. New insights into the function, development, and plasticity of type 2 innate lymphoid cells. Immunol Rev. (2018) 286:74-85. doi: 10.1111/imr.12708

28. Liang HE, Reinhardt RL, Bando JK, Sullivan BM, Ho IC, Locksley RM. Divergent expression patterns of IL-4 and IL-13 define unique functions in allergic immunity. Nat Immunol. (2011) 13:58-66. doi: 10.1038/ni.2182

29. Nussbaum JC, Van Dyken SJ, Von Moltke J, Cheng LE, Mohapatra A, Molofsky $\mathrm{AB}$, et al. Type 2 innate lymphoid cells control eosinophil homeostasis. Nature. (2013) 502:245-8. doi: 10.1038/nature12526

30. Ealey KN, Moro K, Koyasu S. Are ILC2s Jekyll and Hyde in airway inflammation? Immunol Rev. (2017) 278:207-18. doi: 10.1111/imr.12547

31. Bartemes KR, Iijima K, Kobayashi T, Kephart GM, Mckenzie AN, Kita H. IL-33-responsive lineage- CD25+ CD44(hi) lymphoid cells mediate innate type 2 immunity and allergic inflammation in the lungs. J Immunol. (2012) 188:1503-13. doi: 10.4049/jimmunol.1102832

32. Silver JS, Kearley J, Copenhaver AM, Sanden C, Mori M, Yu L, et al. Inflammatory triggers associated with exacerbations of COPD orchestrate plasticity of group 2 innate lymphoid cells in the lungs. Nat Immunol. (2016) 17:626-35. doi: 10.1038/ni.3443

33. Chen H, Sun H, You F, Sun W, Zhou X, Chen L, et al. Activation of STAT6 by STING is critical for antiviral innate immunity. Cell. (2011) 147:436-46. doi: 10.1016/j.cell.2011.09.022

34. Doherty TA, Khorram N, Sugimoto K, Sheppard D, Rosenthal P, Cho JY, et al. Alternaria induces STAT6-dependent acute airway eosinophilia and epithelial FIZZ1 expression that promotes airway fibrosis and epithelial thickness. $J$ Immunol. (2012) 188:2622-9. doi: 10.4049/jimmunol.1101632

35. Moro K, Kabata H, Tanabe M, Koga S, Takeno N, Mochizuki M, et al. Interferon and IL-27 antagonize the function of group 2 innate lymphoid cells and type 2 innate immune responses. Nat Immunol. (2016) 17:76-86. doi: $10.1038 /$ ni.3309

36. Ban GY, Pham DL, Trinh TH, Lee SI, Suh DH, Yang EM, et al. Autophagy mechanisms in sputum and peripheral blood cells of patients with severe asthma: a new therapeutic target. Clin Exp Allergy. (2016) 46:48-59. doi: $10.1111 /$ cea.12585

37. Cavagnero K, Doherty TA. Cytokine and lipid mediator regulation of group 2 innate lymphoid cells (ILC2s) in human allergic airway disease. J Cytokine Biol. (2017) 2:116. doi: 10.4172/2576-3881.1000116

38. Barber GN. STING: infection, inflammation and cancer. Nat Rev Immunol. (2015) 15:760-70. doi: 10.1038/nri3921

39. Ray A, Raundhal M, Oriss TB, Ray P, Wenzel SE. Current concepts of severe asthma. J Clin Invest. (2016) 126:2394-403. doi: 10.1172/JCI84144

40. Benmerzoug S, Rose S, Bounab B, Gosset D, Duneau L, Chenuet P, et al. STING-dependent sensing of self-DNA drives silica-induced lung inflammation. Nat Commun. (2018) 9:5226. doi: 10.1038/s41467-018-07425-1
41. Liu D, Wu H, Wang C, Li Y, Tian H, Siraj S, et al. STING directly activates autophagy to tune the innate immune response. Cell Death Differ. (2018) 26:1735-49. doi: 10.1038/s41418-018-0251-z

42. Gui X, Yang H, Li T, Tan X, Shi P, Li M, et al. Autophagy induction via STING trafficking is a primordial function of the cGAS pathway. Nature. (2019) 567:262-6. doi: 10.1038/s41586-019-1006-9

43. Burdette DL, Vance RE. STING and the innate immune response to nucleic acids in the cytosol. Nat Immunol. (2013) 14:19-26. doi: 10.1038/ni.2491

44. Abe T, Barber GN. Cytosolic-DNA-mediated, STING-dependent proinflammatory gene induction necessitates canonical NF- $\mathrm{KB}$ activation through TBK1. J Virol. (2014) 88:5328-41. doi: 10.1128/JVI.00037-14

45. Kim JA, Park SK, Seo SW, Lee CH, Shin OS. STING is involved in antiviral immune response against VZV infection via the induction of type I and III IFN pathways. J Invest Dermatol. (2017) 137:2101-9. doi: 10.1016/j.jid.2017.03.041

46. Dunphy G, Flannery SM, Almine JF, Connolly DJ, Paulus C, Jønsson KL, et al. Non-canonical activation of the DNA sensing adaptor STING by ATM and IFI16 mediates NF-KB signaling after nuclear DNA damage. Mol Cell. (2018) 71:745-60.e745. doi: 10.1016/j.molcel.2018.07.034

47. Gimeno Brias S, Marsden M, Forbester J, Clement M, Brandt C, Harcourt $\mathrm{K}$, et al. Interferon lambda is required for interferon gamma-expressing NK cell responses but does not afford antiviral protection during acute and persistent murine cytomegalovirus infection. PLoS ONE. (2018) 13:e0197596. doi: 10.1371/journal.pone.0197596

48. Lee AJ, Ashkar AA. The dual nature of type I and type II interferons. Front Immunol. (2018) 9:2061. doi: 10.3389/fimmu.2018.02061

49. Raundhal M, Morse C, Khare A, Oriss TB, Milosevic J, Trudeau J, et al. High IFN- $\gamma$ and low SLPI mark severe asthma in mice and humans. J Clin Invest. (2015) 125:3037-50. doi: 10.1172/JCI80911

50. Canesso MCC, Lemos L, Neves TC, Marim FM, Castro TBR, Veloso É, et al. The cytosolic sensor STING is required for intestinal homeostasis and control of inflammation. Mucosal Immunol. (2018) 11:820-34. doi: $10.1038 / \mathrm{mi} .2017 .88$

51. Ricardo-Gonzalez RR, Van Dyken SJ, Schneider C, Lee J, Nussbaum JC, Liang HE, et al. Tissue signals imprint ILC2 identity with anticipatory function. Nat Immunol. (2018) 19:1093-9. doi: 10.1038/s41590-018-0201-4

52. Luksch H, Stinson WA, Platt DJ, Qian W, Kalugotla G, Miner CA, et al. STING-associated lung disease in mice relies on $\mathrm{T}$ cells but not type I interferon. J Allergy Clin Immunol. (2019) 144:254-266.e258. doi: 10.1016/j.jaci.2019.01.044

53. Maringer K, Fernandez-Sesma A. Message in a bottle: lessons learned from antagonism of STING signalling during RNA virus infection. Cytokine Growth Factor Rev. (2014) 25:669-79. doi: 10.1016/j.cytogfr.2014.08.004

54. Sun L, Xing Y, Chen X, Zheng Y, Yang Y, Nichols DB, et al. Coronavirus papain-like proteases negatively regulate antiviral innate immune response through disruption of STING-mediated signaling. PLOS ONE. (2012) 7:e30802. doi: 10.1371/journal.pone.0030802

55. Zhou P, Tachedjian M, Wynne JW, Boyd V, Cui J, Smith I, et al. Contraction of the type I IFN locus and unusual constitutive expression of IFN- $\alpha$ in bats. Proc Natl Acad Sci USA. (2016) 113:2696-701. doi: 10.1073/pnas.15182 40113

56. Xie J, Li Y, Shen X, Goh G, Zhu Y, Cui J, et al. Dampened STING-dependent interferon activation in bats. Cell Host Microbe. (2018) 23:297-301.e294. doi: 10.1016/j.chom.2018.01.006

57. Cavagnero K. Cyclic-di-GMP induces STING-dependent ILC2 to ILC1 shift during innate type 2 lung inflammation. bioRxiv. (2020). doi: $10.1101 / 2020.10 \cdot 19.345850$

Conflict of Interest: The authors declare that the research was conducted in the absence of any commercial or financial relationships that could be construed as a potential conflict of interest.

Copyright () 2021 Cavagnero, Badrani, Naji, Amadeo, Leng, Lacasa, Strohm, Renusch, Gasparian and Doherty. This is an open-access article distributed under the terms of the Creative Commons Attribution License (CC BY). The use, distribution or reproduction in other forums is permitted, provided the original author(s) and the copyright owner(s) are credited and that the original publication in this journal is cited, in accordance with accepted academic practice. No use, distribution or reproduction is permitted which does not comply with these terms. 